Running head: Aphasia in Persian

\title{
Aphasia in Persian: Implications for cognitive models of lexical processing
}

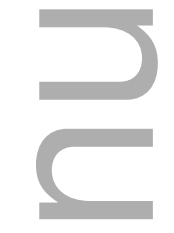

Mehdi Bakhtiar*1, Reyhane Jafary², Brendan Weekes ${ }^{1}$

${ }^{1}$ Laboratory for Communication Science, Division of Speech and Hearing Sciences, University of Hong Kong, Hong Kong ${ }^{2}$ Speech Therapy Department, Tehran University of Medical Sciences, Tehran, Iran

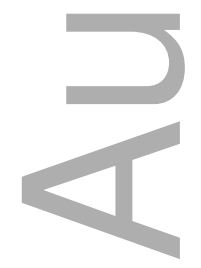

This is the author manuscript accepted for publication and has undergone full peer review but has not been through the copyediting, typesetting, pagination and proofreading process, which may lead to differences between this version and the Version of Record. Please cite this article as doi: $\underline{10.1111 / \text { inp.12095 }}$

This article is protected by copyright. All rights reserved 
* Corresponding Author: Mehdi Bakhtiar, Laboratory for Communication Science, Division of Speech and Hearing Sciences, University of Hong Kong, Hong Kong (e-mail:

bakhtiar@connect.hku.hk).

-
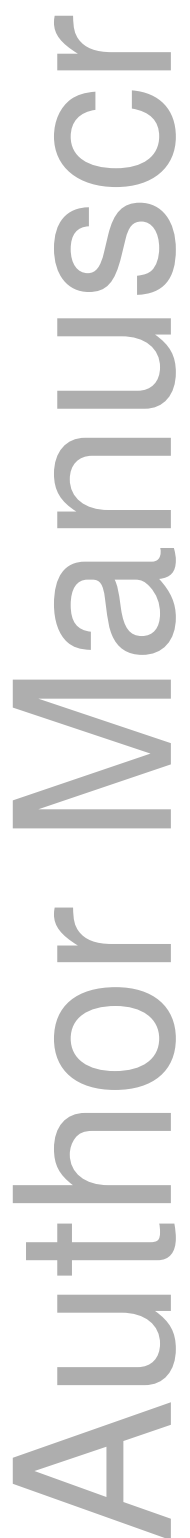

This article is protected by copyright. All rights reserved 


\title{
Received Date: 01/08/2015
}

\section{Revised Date: 22/11/2015}

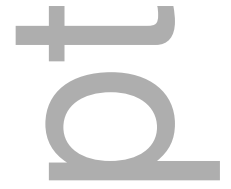

\section{Accepted Date: 30/11/2015 \\ Article Type: Research Paper \\ Aphasia in Persian: Implications for cognitive models of lexical processing}

\begin{abstract}
Current models of oral reading assume that different routes (sublexical, lexical and semantic) mediate oral reading performance and reliance on different routes during oral reading depends on the characteristics of print to sound mappings. Studies of single cases of acquired dyslexia in aphasia have contributed to the development of such models by revealing patterns of double dissociation in object naming and oral reading skill that follow brain damage in Indo-European and Sino-Tibetan languages. Print to sound mapping in Persian varies in transparency because orthography to phonology translation depends uniquely on the presence or absence of vowel letters in print. Here a hypothesis is tested that oral reading in Persian requires a semantic reading pathway that is independent of a direct non-semantic reading pathway, by investigating whether Persian speakers with aphasia show selective impairments to object naming and reading aloud. A sample of twenty one Persian speakers with aphasia ranging in age from 18 to 77 (mean= 53, SD=16.9) were asked to name a same set of 200 objects and to read aloud the printed names of these objects in different sessions. As an additional measure of sublexical reading, patients were asked to read aloud 30 nonword stimuli. Results showed that oral reading is significantly more preserved than object naming in Persian speakers with aphasia. However, more preserved object naming than oral reading was also observed in some cases. There was a moderate positive correlation between picture naming and oral reading success $(\mathrm{p}<0.05)$. Mixed-effects logistic regression revealed that word frequency, AoA and imageability predict success across both tasks and there is an interaction between these variables and orthographic transparency in oral reading. Furthermore, opaque words were read less accurately than transparent words. The results reveal different
\end{abstract}


patterns of acquired dyslexia in some cases that closely resemble phonological, deep and surface dyslexia in other scripts - reported here in Persian for the first time.

Keywords; Aphasia, Persian, orthographic transparency

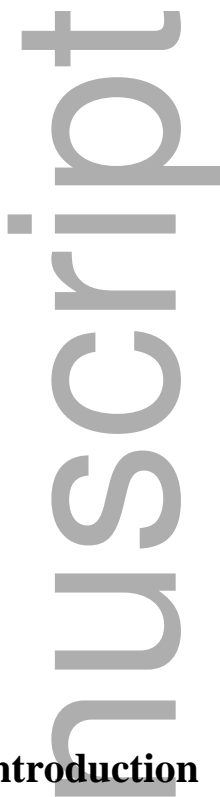

Cognitive neuropsychological studies of acquired anomia and dyslexia have informed theoretical models of spoken word production and oral reading in several languages. In the case of object naming, models assume that phonological output is supported by mechanisms that are recruited during successive stages starting from object form recognition followed by conceptual identification, lemma selection and word form (phonological) encoding (Levelt, 1999; Levelt, Roelofs, \& Meyer, 1999). Models of oral reading by contrast typically assume at least two pathways are available for phonological output: one that proceeds via conceptual identification (the semantic route) and the other that proceeds without semantic mediation (the direct route). Studies of aphasia in Chinese, English, French, German, Hebrew, Italian, Japanese, Korean, Portuguese, Slovakian and Spanish tends to support these assumptions, suggesting some degree of cross-linguistic similarity in cognitive neuropsychological processing of objects and words (Weekes, 2005, 2012).

Cross linguistic studies also reveal that models of oral reading may also differ according to whether the direct route requires division into a lexical and a sublexical pathway. For Indo-European languages, it is assumed by virtually all theorists that a sublexical route is available to convert the sublexical units of print e.g. graphemes into phonemes for articulation during oral reading either by using a rule based process 
(e.g. Coltheart, 1978) or by analogical processes (e.g. Glushko, 1979). The classic dual route computational (DRC) model makes this assumption that skilled oral reading is supported by three pathways of semantic, lexical and sublexical routes (M. Coltheart, Curtis, Atkins, \& Haller, 1993; M. Coltheart, Rastle, Perry, Langdon, \& Ziegler, 2001). In this model (Coltheart et al., 2001), the sublexical pathway is independent of a direct, lexical route, which is available for reading words that do not conform to typical rules of grapheme to phoneme correspondence in the language. For example to read correctly the words yacht in English, baca in Spanish and macchina in Italian requires lexical knowledge about the word that is unique in terms of atypical phonological output assigned at the level of the individual phoneme, rime or syllable. This knowledge is referred to as lexical because it requires memory for word pronunciation that is not marked in print by the assignment of sublexical rules or analogies. Such a division of labour between lexical and sublexical pathways is not universally accepted however. Some theorists argue it is not necessary to divide nonsemantic reading into lexical and non-lexical pathways, as the lexical representations are not assumed. Most Parallel Distributed Processing (PDP) computational models make this assumption (Plaut, McClelland, Seidenberg, \& Patterson, 1996; Seidenberg $\&$ McClelland, 1989). Another class of computational model called the CDP+ model (Perry, Ziegler, \& Zorzi, 2007) assumes a direct lexical pathway (similar to DRC) and a connectionist sublexical pathway with units of graphemes and phonemes organized into onsets and codas.

Almost all computational models of oral reading are developed to explain the underlying mechanism of oral reading based on the features of English orthography (for a review see Perry, et al., 2007). It is typically assumed by researchers of reading in other languages that such models can explain patterns of oral reading in a diverse range of languages. Surprisingly this is true for languages with non-alphabetic writing systems including Chinese (see Yang, McCandliss, Shu, \& Zevin, 2009, for a PDP model of reading in Chinese) and Hebrew (See Velan, Frost, Deutsch, \& Plaut, 2005 for a PDP model of reading in Hebrew). This assumption extends to studies of acquired dyslexia in a variety of languages with a diverse range of scripts including Arabic, Greek, Hebrew, Hindi, Japanese, Korean and others (Beland \& Mimouni, 2001; Weekes, 2005, 2012). However, this assumption can be challenged (see for example Coltheart et al. 2001; Weekes, Yin, Su \& Chen, 2006; Yin \& Weekes, 2003). The reason is that some languages do not encode some phonemes in script making 
grapheme to phoneme rules redundant e.g. Hebrew and other languages can only be read using lexical processes e.g. Chinese (with the possible exception of a handful of characters that do not contain lexical representation at all, see Law, Wong, Yeung, and Weekes, 2008) making a sublexical route unnecessary. Thus, extant models may not capture the uniqueness of reading and reading disorders across the many types of script used in the worlds' languages (Frost, 2012).

Turning to the question of reading in cases of individual speakers with aphasia it is universally accepted that such cases evidence patterns of dissociation in semantic and nonsemantic oral reading of written words and indeed it is agreed by all theorists that different patterns of acquired dyslexia provide constraints on the development of computational models of oral reading (Coltheart et al., 2001; Plaut et al., 1996). The evidence supports the view that oral reading in different language families including Semitic (Hebrew) and Sino-Tibetan (Chinese) requires at a minimum two pathways: a semantic and a direct-nonsemantic pathway (Weekes, 2005; 2012). The question whether direct non-semantic reading requires analysis of fine-grained sublexical units i.e. mapping print and sound, seems to be language or more precisely script specific. The evidence for this assertion will be discussed further below.

Overall, three types of acquired dyslexia have been described in the literature. A patient is said to have surface dyslexia when they can read nonwords and regular words correctly while having a deficit in reading low frequency irregular words. In fact, cases of surface dyslexia tend to regularize irregular words e.g. pint which are read to rhyme with mint (Funnel 2000), presumably because they rely on a nonlexical route for word reading. In cases of phonological dyslexia there is a deficit in reading nonwords (pronounceable stimuli with no meaning) with relative sparing for reading familiar words and no evidence of regularization errors. Such cases tend to lexicalise nonwords e.g. milp read as milk presumably because they rely on lexical processes. Finally, deep dyslexia is characterized by poor performance in reading both words and nonwords characterized by semantic, visual, and mixed errors. These errors are assumed to result from reliance on the semantic pathway for reading. Dissociations in patterns of acquired dyslexia across languages support a dual route framework of reading at a minimum. Furthermore reports of intact reading of irregular words that are not understood supports the assumption of a direct lexical pathway that separate from a direct lexical pathway for oral reading in English (see Coltheart et al. 2001). 
In addition to constraining models of oral reading, studies of patients with aphasia can inform models of spoken word production (Nickels \& Howard, 1995). Such studies typically examine effects of lexico-semantic variables (e.g. frequency, AoA and imageability) on picture (actions and objects) naming performance to address how the normal mechanisms of spoken word production dissociate across patients. Nickels and Howard (1995) were the first to study the effects of AoA, object manipulability, frequency, familiarity, imageability, concreteness, length and visual complexity of a depicted object on picture naming in aphasia in English. Their results showed that for one sub-group of cases AoA, manipulability and word length were predictive of performance whereas in another subgroup imageability was a significant predictor. However, as expected for group studies of aphasia (Caramazza, 1984), the predictor variables did not consistently predict performance across individual cases. Similarly in Spanish, Cuetos, Aguado, Izura, and Ellis (2002) found that although name AoA, concept familiarity, frequency and visual complexity predicted picture naming, variables did not consistently predict the performance of any individual (see also Weekes et al., 1998 who came to a similar conclusion for Chinese speakers). Studies with a focus on individual cases of aphasia are often more informative for models of spoken word production and oral reading. For example, Hirsh and Ellis (1994) found words learned earlier were more accurately retrieved in different tasks including spoken and written naming and reading aloud in a patient with nonfluent aphasia. Using logistic multiple regression they found that AoA was a better predictor than highly correlated variables such as frequency and rated imageability. Barry and Gerhand (2003) reported a significant effect of AoA and concreteness on oral reading accuracy and word comprehension in a patient with deep dyslexia. They argued that the concreteness effect is an index of semantic activation for accessing phonological output during oral reading in English (Barry \& Gerhand, 2003). Law et al. (2008) reported effects of rated AoA and orthography to phonology consistency as well interaction between these variables on oral reading in a case of acquired dyslexia in Chinese and argued these effects indicated reading via a non-semantic reading pathway in Chinese (see also Weekes et al. 1997; Weekes, 2012). Using linear mixedeffect modelling which considers individual differences across cases as well as fixed effects of item characteristics, Crepaldi, Che, Su, and Luzzatti (2012) found significant independent effects of word frequency and AoA on reading together with significant effects of word frequency and imageability on picture naming. The power 
of this analysis is that for each case it is possible to know whether the variables that impact on lexical processing depend on task i.e. picture naming or oral reading. This adds to the value of case data by revealing dissociations in performance case by case.

We know nothing about the functional architecture of the lexical-semantic system supporting spoken word production and oral reading in Persian. One reason to explore this question concerns the unique mappings between spoken (Persian) and the written (Arabic) script. Although this arbitrary pairing is not unique historically (e.g. Albanian), Persian together with related languages Urdu (spoken in Pakistan) and Pashto (spoken in Afghanistan) are the only Indo-European languages that use a modified version of a Semitic script (Arabic) (Mirdehghan, 2010). Another unique feature of written Persian is the high degree of grapheme to phoneme consistency, where each grapheme corresponds to only one phoneme (Bakhtiar \& Weekes, 2015). Despite the potential consistency between graphemes and phonemes in text, literate Persian speakers typically read words that are written without diacritic markings for some vowels (as in Semitic languages) making most text relatively opaque for adult readers. For example, three short vowels $(\mathrm{a}, \mathrm{e}, \mathrm{o})$ in Persian are marked in print for the benefit of beginning readers only. The spelling of words containing these phonemes can thus be considered opaque (e.g. srd $\rightarrow$ /sard/, "cold") for adult readers. On the other hand, written words with long vowels $(\hat{a}, \mathrm{u}, \mathrm{i})$ do have distinct letter formats that are depicted in adult text and can therefore be considered as transparent ( $\mathrm{sib} \rightarrow$ /sib/, "apple"). Orthographic depth is therefore missing for some words but not for others. Significant effects of orthographic transparency on oral reading in aphasia have been established for a wide range of Indo-European, Semitic and Sino-Tibetan languages and characterized as surface alexia/dyslexia in most cases. This provides a rationale for investigating potential effects on the reading of Persian speakers with aphasia.

The primary aim of this study is to test the hypothesis orthographic depth has an effect on oral reading performance in Persian speakers with aphasia as observed in other languages (Chinese, English and Hebrew). As shown by Crepaldi et al. (2012), this can best be achieved using mixed modeling which has the advantage over typical regression by analysing random effects including intra-individual characteristics such as age, lesion and aphasia type. Also following Crepaldi et al. (2012), we compared oral reading and picture naming performance to test the hypothesis that item variables would have differential effects according to task. We expected acquired impairment in reading (dyslexia) to be co-incident with impaired picture naming (anomia) and a 
dissociation whereby impairments would be observed with preserved comprehension. We also expected reading of opaque words to be more affected than transparent words by aphasia following studies of reading in Persian (Baluch, 1993; Baluch \& Shahidi, 1991; Rahbari \& Senechal, 2009, 2010) including developmental dyslexia (Baluch \& Danaye-Tousi, 2007; Pouretemad, Khatibi, Zarei, \& Stein, 2011). As in other Semitic scripts, we expected reading disorders to resemble the patterns of deep, phonological and surface dyslexia already reported in Arabic and Hebrew (Beland \& Mimouni, 2001; Friedman \& Haddad, 2015).

\section{Methodology}

\subsection{Subjects}

Twenty-one brain-injured Persian-speakers ranging in age from 18 to 77 (mean= 53, $\mathrm{SD}=16.9)$ participated. The patients suffered from aphasia due to CVA (16 cases), head trauma (three cases), brain infection (one case) and frontotemporal dementia (one case). The lesion onset time ranged from 2 to 168 months (mean=50, SD=41). The number of years of formal education ranged from 3 to 18 years. Participants were assessed using a reduced form (Bedside) version of the Persian (P-WAB-1) adapted from the Western Aphasia Battery and then classified into two groups of fluent (16) and non-fluent cases (5) based on an overall score including the fluency score of the Persian WAB (Nilipour, Pourshahbaz, \& Ghoreyshi, 2014). Table 1 summarises demographic information for all participants together with proportion of naming and reading accuracy.

\subsection{Stimuli and Procedure}

The stimuli were 200 colored pictures of objects from the Snodgrass and Vanderwart (1980) set and the written transcriptions of the object names. All stimuli were standardized in Persian based on psycholinguistic variables including the concept familiarity, word frequency, rated age of acquisition (AoA), word length (i.e. number of phonemes and letters), visual complexity, image agreement, name agreement, imageability and degree of transparency (DT) between orthography and phonology (Bakhtiar, Nilipour, \& Weekes, 2013). The DT was computed by dividing the number of a word's letters by the number of its phonemes. The values of DT ranged from 0.5 (the most opaque) to 1 (the most transparent) (Mean $=0.8, \mathrm{SD}=0.12)$. A total number of 44 words $(22 \%)$ were completely transparent $(\mathrm{DT}=1)$ and all others were opaque 
(to different extents) with DT scores ranging between 0.5 and 0.91 . The word and picture naming tasks were presented in two different sessions while task order was counter balanced across the patients. Each item was presented for five seconds and then replaced by a blank page lasting for two seconds. After this, the next trial began. The stimuli were presented in three blocks with two breaks in-between. However, if a patient felt tired or was reluctant to respond, the experimenter could pause the task.

A nonword reading task was administered to assess the integrity of the nonlexical pathway. Nonwords cannot rely on lexico-semantic information for correct pronunciation and therefore reflect operation of the non-lexical grapheme to phoneme pathway. For nonword reading, 30 nonwords varying in letter length from two to eight letters (mean=4.6) were created by changing consonants and vowels of words to create meaningless stimuli. Thirty six unimpaired speakers were asked to read nonwords aloud to see if they could articulate a response as judged by native Persian speakers. All participants were able to read every nonword accurately in less than 5 seconds. This result shows that all control participants were able to articulate a novel spoken utterance using grapheme to phoneme correspondences. Responses to object pictures and written words were simultaneously registered and audio-recorded by the examiner and later classified into semantic, formal, visual-perceptual, mixed, unrelated and neologisms based on the classification proposed by Dell et al. (1997). Based on this classification, responses that were synonyms, category coordinates, category subordinates, and category superordinates or associates of the target word are semantic errors. Responses sharing the same initial and/or final phonemes with the target, or shared similar phoneme at the same position with the target or more than one common phoneme in any positions are formal/visual errors. Some formal reading errors were substitutions of the target with orthographically similar words and these were labeled visual errors. For picture naming, responses with visual similarity to the target were called visual-perceptual errors. Responses meeting the criteria for both semantic and formal errors were called mixed errors. Responses that did not meet any criteria for semantic or formal error included neologisms and perseverations classified according to their relation to target words as semantic, formal or unrelated errors. 


\section{Results}

There was a moderate positive correlation between picture naming and oral reading, which was statistically significant both within subjects $(r=0.54, p<.05)$ and items $(\mathrm{r}=0.60, \mathrm{p}<.0001)$. The proportion of correct naming responses for picture naming and oral reading were compared using Wilcoxon Signed Ranks Test and the results showed that oral reading was significantly better than picture naming $(Z=-3.72, p<$ .0001). To examine dissociations between naming and reading, McNemar's test was applied to test individual differences for each case. Results showed that 17/21 (85\%) patients had significantly better performance in reading compared to naming, one patient showed a trend towards better reading than naming (i.e. Case 1) $\chi^{2}(1)=2.85$, $\mathrm{p}=.09$, two cases showed no significant difference between reading and naming (i.e. Cases 10 and 21) $\chi^{2}(1)=2, p>.05$, and one case (i.e. Case 5) showed significantly better naming compared to reading $\chi^{2}(1)=10.23, \mathrm{p}<.01$, although notably his naming performance was rather poor (41\% accuracy) (see also Figure 1 and Table 1). In terms of the lesion side the results showed that generally patients with the left hemisphere damage (LHD) versus to the patients with right hemisphere damage (RHD) showed lower performance both in word reading (80\% versus 93\%) and picture naming ( $52 \%$ versus $83 \%$ ) tasks. Furthermore, the dissociation between naming and reading tasks is higher for LHD patients (\%28) than RHD ones (10\%) indicating that brain lesion in LH is more detrimental for object naming than oral reading. Although one patient with bilateral brain damage showed a high performance in picture naming $(70 \%)$, however, it needs to be notified that her main lesion was more on the right hemisphere. In terms of aetiology, it can be inferred from the data that patients with CVA (specifically in left hemisphere) show lower performance than patients with head trauma (specifically in right hemisphere) across both reading and naming tasks (see Table 1). Moreover, it is noticeable that a patient with Frontotemporal dementia (FTD) had dramatically lower performance in picture naming (26\%) than word reading (87\%), while a patient with brain infection had comparably low performance in picture naming (24\%) and word reading (29\%).

The distribution of naming errors in the reading and naming task were compared using Wilcoxon signed rank sum test given that are not normally distributed. The result showed that semantic errors $(Z=4.0, p<.001)$ and unrelated errors $(Z=2.5, p$ $<.05)$ were more frequent in naming than in reading, while formal errors were 
significantly more frequent in reading compared to naming $(Z=-3.23, p<.01)$. The distributions of mixed errors and neologisms were not different between reading and naming tasks. Table 2 shows distribution of errors in naming and reading. Nonword reading accuracy for 10 cases is reported in Table 1 . The range varied from $0 \%(0 / 30)$ to $97 \%$ (29/30) accuracy with some cases showing more impaired nonword reading than word reading (Cases 5, 13, 14, 16 \& 17). Overall, the errors in nonword reading were no-response errors (44\%), lexicalization errors i.e. reading nonwords as real words $(8 \%)$ and visual errors $(4 \%)$.

<Insert Tables $1 \& 2$ and Figure 1 here>

Correlations between item characteristics and accuracy for naming and reading are shown in Table 3. AoA has the highest (negative) correlation with naming and reading accuracy. Notably, concept familiarity shows high correlations with many psycholinguistic variables. A diagnostic test of collinearity given by Stata (condition number), revealed a high collinearity between variables (condition number $=60$ ). This degree of intercorrelation between variables makes it difficult to isolate significant independent effects of each variable using standard regression models and can lead to unstable inference (Baayen, 2008). To minimise this problem, two steps were taken (1) variables were centred on their means and (2) familiarity was removed from the list of variables as it was highly correlated with all variables (i.e. rated AoA, imageability and frequency) (See Table 3). Collinearity was diminished significantly (condition number $=3$ ). A mixed-effect logistic (MEL) regression was then applied because MEL regression can consider variability at both the participant and item levels using Stata software.

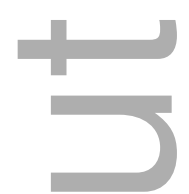

$<$ Insert Table 3 here>

3.1 Mixed effects logistic regression

\subsubsection{Oral reading}

In the first MEL analysis, a stepwise MEL regression model was developed to predict reading accuracy. Individual differences were entered first including aphasia type, chronological age, post-onset duration (i.e. months) and education (i.e. years). Then in the second step the centred psycholinguistic variables related to each task were 
entered one by one, and the nested models were compared through a likelihood ratio test (LRT) based on procedures recommended by Statistical Consulting Group of the University of California, Los Angeles (www.ats.ucla.edu/stat/stata/ado/analysis/). Four cases with complete accuracy (100\%) in oral reading were excluded from MEL. The first step revealed significant effects of aphasia type i.e. fluent cases performed better than non-fluent cases; older cases performed better than younger cases; postonset duration (i.e. longer the better); and education (higher the better) on oral reading accuracy. In the second model, word frequency values for test items were entered into the model followed by other fixed variables (rated imageability, AoA, number of letters and DT values). Contrasting Model 1 (nested in Model 2), including participant values, and Model 2, including the participant values plus word frequency values, using LRT produced a significant result $\chi^{2}(1)=62.0, \mathrm{p}<.00001$, which implies that adding word frequency values significantly improved the model fit (more frequent words were read more accurately). Adding imageability next into the model also improved the model fit $\chi^{2}(1)=17.0, p<.00001$ with imageable words named more accurately. AoA was added last into the model and also improved the fit significantly $\chi^{2}(1)=14.0, p<.001$ i.e. early acquired words read more accurately than late acquired words. Number of letters did not significantly improve the model fit $\chi^{2}(1)=0.2, p=$ 0.7. Finally, degree of transparency (DT) significantly improved the model fit $\chi^{2}(1)$ $=14, \mathrm{p}<.001$ with transparent words read more accurately than opaque words (see Table 4).

<Insert Figure 2 here>

DT interacted significantly with AoA, frequency and imageability (see Table 4 and Figure 2). Words with higher DT (i.e. more transparent words) were read more accurately when frequency and imageability values increase, while these effects are reduced for the most opaque words (with lowest DT values). Early acquired words with lower DT values (i.e. more opaque) were named more accurately than late acquired opaque words and the effect of AoA effect was reduced as words become more transparent (Table 4 and Figure 2). Additional analysis considered the interaction between chronological age and rated AoA, to test if the effect of AoA can be explained by cumulative frequency as hypothesised by some theorists (Zevin \& Seidenberg, 2002). There was no significant improvement to the fit of the model by 
adding this interaction term $\chi 2(1)=0.001, \mathrm{p}=0.98$.

\subsubsection{Picture naming}

In the next MEL analysis of picture naming accuracy, results show significant effects of aphasia type (i.e. fluent better than non-fluent), age (younger the better), post-onset duration (i.e. longer the better) and education (higher the better), on naming accuracy. Significant predictor variables were name agreement, image agreement, visual complexity, word frequency, AoA, imageability and number of phonemes. Name agreement was added first into Model 1 (individual differences) to create Model 2. Contrasting Model 1 and Model 2 using LRT produced a significant result $\chi^{2}(1)=$ 93.0, $\mathrm{p}<.00001$, i.e. pictures with higher name agreement were named more accurately. Image agreement was entered into the model and significantly improved the model fit $\chi^{2}(1)=12.0, \mathrm{p}<.001$, showing that objects with more image agreement were named more accurately. Visual complexity (VC) did not improve the model fit significantly $\chi^{2}(1)=0.3, p=.6$, although imageability $\chi^{2}(1)=36, p<.00001$, word frequency $\left.\chi^{2}(1)=42.0, p<.00001\right)$, number of phonemes $\chi^{2}(1)=47.0, p<.00001$ AoA $\chi^{2}(1)=29.0, p<.00001$, did improve model fit. As for oral reading, there was no additional contribution from the addition of an interaction between chronological age of the patients and AoA of the word. Therefore, the result showed that adding this interaction could not improve the model fit at al $\chi^{2}(1)=-0.06, p=1$.

\subsubsection{Final models}

The final models included the random effect of participants and items (Model A) were compared to a model with only random effects for items (Model B) and a model with only random effect of participants (Model C). These were compared to determine whether inclusion of the random effects from participants and from items could explain significant variance in word and picture naming. For oral reading, LRT revealed a significant difference between Models A and B $\chi 2(1)=17, p<.00001$, confirming a significant random effect from participants. However, the difference between Model A and Model C using LRT was not significant $\chi 2(1)=-0.2, p=1$, which shows that testing the random effect of items was not important for these data. Table 4 shows the final model for predictors of oral reading among Persian speakers with aphasia with the report for the random effect of subjects. For picture naming, the LRT indicated a significant difference between model A and B $\chi^{2}(1)=62.0, \mathrm{p}<$ 
.00001 , justifying the random effect of participants. However, the difference between model A and model $\mathrm{C}$ using LRT was not significant $\chi 2(1)=0.4, p=0.8$, indicating that taking the random effect of items into account was not important. Table 5 shows the final model for picture naming with the report of random effect of participants.

\section{$<$ Insert Tables 4 \& 5 here>}

\section{Discussion}

We expected that (1) dissociations between picture naming and oral reading would be observed in Persian speaking patients with aphasia; (2) greater impairment when reading words with opaque spellings; and (3) psycholinguistic variables would differentiate picture naming and oral reading. Overall, the group analysis found that oral reading is significantly better preserved than picture naming for most cases consistent with other reports studies in languages such as Chinese and English (Breen \& Warrington, 1995; Law, Wong, \& Chiu, 2005; Weekes, Chen, \& Gang, 1997). This extends this observation to an Indo-European language with a Semitic script. It is likely that although the writing system adopted by Persian speakers is parasitic on the spoken (Persian) language, adopting the Arabic script engages neuro-cognitive processes that are independent of spoken word processing and can therefore survive brain injury and severe aphasia. That is not to say however that dissociation between

oral reading and picture naming necessarily favors reading over speaking. At least one case showed the reverse dissociation whereby picture naming was better (not perfect) than oral reading performance. At the very least, the patterns of dissociation shown by these cases suggests that different pathways are available to access the phonological output lexicon in Persian (see Figure 3): a semantic route for picture naming and a direct, non-semantic route for mapping orthography to phonology (OP) during oral reading. Note that oral reading may tap into the semantic route but the reverse i.e. spoken word production tapping into the direct non-semantic route is unlikely. The pattern of results can be interpreted as showing that for most cases of aphasia in Persian, the direct non-semantic reading route/s is relatively intact allowing the correct mapping between orthography to phonology correspondences that require no semantic input. By contrast, picture naming is relatively poor because the semantic route is impaired (see Figure 3 for a pictorial depiction).

$<$ Insert Figure 3 here> 
Further evidence of dissociation between the direct and semantic pathways to lexical access can be found by comparing the pattern of naming and reading errors, showing that semantic errors are more frequent in picture naming while formal/visual errors are more frequent in oral reading. The prevalence of formal errors in reading indicates reliance on the direct non-semantic route/s (which could be either lexical or sublexical or both cf. Coltheart et al., 2001) presumably due to some deficit in the semantic route. Furthermore although the effect of psycholinguistic factors had very similar impact on picture naming and oral reading (i.e. significant effects of AoA, frequency and imageability in both tasks), word length (number of phonemes) had a significant effect on picture naming only. An effect of word length on picture naming has been related to the stage of phonological encoding or articulation (Alario et al., 2004), which may indicate that phonological retrieval (from semantic representations) for longer words is more difficult (Cuetos et al., 2002; Nickels \& Howard, 1995). The null effect of word length (i.e. number of letters) on oral reading accuracy cannot be explained with certainty. Word length effects in oral reading are considered an index for nonlexical reading. Therefore, one speculation is that impaired reading in Persian is less dependent on the nonlexical reading route than other Indo-European languages. This may be because Persian has a relatively opaque orthography. Based on the recent literature showing an inhibitory effect of increasing word length on reading only for words longer than seven or eight letters in English and Spanish (Gonzalez-Nosti, Barbon, Rodriguez-Ferreiro, \& Cuetos, 2014; New, Ferrand, Pallier, \& Brysbaert, 2006), we may speculate that effects of length are absent because a majority of word stimuli in this study are shorter than eight letters (93\%). However, we caution against over interpretation of the null finding.

Image agreement and name agreement (marginal effect) predicted picture naming in aphasia consistent with results from a study of young Persian speaking adults (Bakhtiar et al., 2013). The effect of image agreement indicates that objects matching prototypical mental images less well are named less accurately in aphasia and has been related to object recognition (Alario et al., 2004). Presumably this is a potential locus of anomia in Persian as in English and Chinese speaking patients with aphasia (Humphreys, Riddoch, \& Quinlan, 1988; Law, Weekes, Yeung, \& Chiu, 2009) and mandates the assessment of object recognition in such patients i.e. by using the Birmingham Object Recognition Battery (Riddoch \& Humphreys, 1993). The effect of name agreement shows objects with more alternative names are more vulnerable in 
aphasia presumably because they impose more lexical competition at the lemma level in picture naming (Alario et al., 2004). This resonates with models of picture naming and spoken word production (e.g. Levelt et al., 1999) and with the performance of Arabic, Chinese, English, Spanish and bilingual speakers with aphasia (Kong, Abutalebi, Lam, \& Weekes, 2014).

Highly imageable words are retrieved more accurately than low imageable words across both tasks. An imageability effect has been reported on oral reading in aphasia particularly reading of low frequency, irregular words by skilled readers in Chinese and English (both children and adults) and indicates a requirement to access semantic representations during reading aloud (V. Coltheart, Laxon, \& Keating, 1988; Franklin, Howard, \& Patterson, 1995; Strain, Patterson, \& Seidenberg, 1995). Note, however that Monaghan and Ellis (2002) argued that effects of imageabilty (assumed to be a semantic variable) are in fact due to AoA, which is highly correlated with rated imageability (see also Table 3). Independent effects of AoA and frequency were found on both word and picture naming. As these variables are highly correlated some theorists argue that the AoA effect is no more than cumulative frequency or word residence time (Zevin \& Seidenberg, 2002). We found no evidence of an interaction between age and word AoA (Morrison, Hirsh, Chappell, \& Ellis, 2002) as would be expected on this account. Instead we submit the effect of AoA in Persian has a semantic or semantic to phonological locus that can be differentiated from an independent effect of frequency on picture naming and oral reading in Persian (Bakhtiar et al., 2013; Bakhtiar \& Weekes, 2014).

The independent effect of orthographic transparency on impaired oral reading when other lexico-semantic variables (i.e. frequency, imageability and AoA) are controlled is compatible with skilled adult readers (Bakhtiar \& Weekes, 2015) and children with dyslexia in Persian (Baluch \& Danaye-Tousi, 2006). One speculation is that opaque words are less likely to be read aloud correctly using the non-semantic route alone (bypassing the impaired semantic route), whereas transparent words depend less on input from the lexical-semantic route (Bakhtiar \& Weekes, 2015). Positive correlation between oral reading and picture naming performance in Persian speakers with aphasia is consistent with this interpretation and suggests that success in oral reading may be at least partially influenced by severity of impairment in the semantic system (i.e. picture naming), which is more related to reading opaque words. Nonetheless, it is notable that at least four patients showed perfect reading accuracy 
despite minor to moderate impairment to picture naming (see Table 1). It is important to consider that the word stimuli are all names of imageable objects and therefore a less severe semantic impairment may not influence oral reading for these types of words. Future research in patients with both acquired aphasia and dyslexia in Persian may consider presenting transparent and opaque words with wider range of rated imageability or concreteness (i.e. having both concrete and abstract words) to test whether damage to the semantic system affects reading of opaque orthography to phonology mappings.

The significant interactions between DT and AoA, frequency and imageability revealed that more opaque words benefit more from early exposure whereas the more transparent words are less affected by late acquisition and might receive extra benefit from the positive effects of high frequency and imageability (see Figure 2 and Table 4). If, as we assume, AoA has a potential semantic locus during oral reading it may be that late exposure has a detrimental effect on oral reading of opaque Persian words because the mapping between input and output is arbitrary (Monaghan \& Ellis, 2002). Detailed analysis of the patterns of reading deficits across patients found two cases with relatively preserved performance in oral reading but poor performance in nonword reading. Case 13 had remarkably better performance in word reading (91\% accuracy) than nonword reading (10\% accuracy) and Case 16 had a similar outcome (87\% word accuracy and 7\% nonword accuracy). Reading errors were exclusively noresponse errors and formal errors (no semantic errors). Results from these cases may suggest selective damage in assembling phonemes when reading nonwords using a nonlexical pathway. This pattern resembles phonological dyslexia (Coslett, 1991) in Indo-European (English and Spanish) and Semitic languages (Arabic and Hebrew). According to the DRC model, acquired phonological dyslexia is due to damage in the nonlexical reading pathway most likely in grapheme to phoneme correspondence. In our view, the pattern of reading impairment for Cases 13 and 16 reflects a dissociation between the direct lexical and nonlexical pathways, which are both dissociable from a semantic reading pathway (Coltheart et al., 2001). According to PDP triangle models of oral reading (K. Patterson, 2000), phonological dyslexia occurs due to damage in the sublexical pathway, which forces the participant to rely on reading words via the intact semantic system. This could explain performance for Case 13 with relatively good performance in picture naming (77\% accuracy) and preserved semantic system but not Case 16 who shows very poor performance in picture naming (15\% accuracy). 
Three cases had relatively severe problems in oral reading (less than 50\% correct) including Case 5 (26\% accuracy), Case 10 (29\% accuracy) and Case 17 (46\% accuracy). Notably, all of these cases performed poorly in nonword reading, and in fact Case 5 and Case 17 were not able to read nonwords at all. Case 10 could only read about $17 \%$ of nonwords correctly ( 5 out of 30 ). There was evidence that Case 5 read nonwords using a lexical reading pathway as he produced mostly lexicalization errors during nonword reading ( $44 \%$ of stimuli). For example, the nonwords qix, and âkrâš were read aloud as qâšoq (spoon) and âšpazi (cooking). It is important to note also that Case 5 had a relatively low premorbid level of literacy (i.e. Grade 5) and this may account for his impaired reading performance. However, it does not provide a full explanation of his reading impairment, because stimuli had estimated maximum age of acquisition of 8.5 years, which is approximately equal to Grade 3 in the Iranian educational system. Indeed, it is also notable that Case 1 had a lower literacy level (i.e. Grade 3) and yet she had better reading accuracy (55\%) than Case 5 (26\%) indicating that Case 5's lower performance in reading cannot be explained entirely by premorbid level of literacy.

In our view, these cases were all reading via a semantic pathway. Evidence for this assertion comes from the range of semantic errors, formal/visual errors and mixed visual-semantic errors produced (see Tables $2 \& 6$ ). Examples of semantic errors are words يير اهن ketâb pirâhan (book and shirt) that were read as دفتاب daftar and Šlalvâr (notebook and pants) respectively. Mixed visual-semantic errors, for example is the word خرس xers (bear) that was read as خروس xorus (rooster) were also observed. Another example is سوت sut (whistle) read as سوزن suzan (needle). As for visual errors, a word like دكترى setri (kettle) was read as سترن doktor (doctor), and نمكدان namakdân (saltshaker) was read as دندان dandân (tooth) (see Table 6). The errors from these cases are similar to those described as deep dyslexia in relatively deep Indo-European orthographies such as English and French (for a review see Funnel, 2000) and even shallow orthographies such as Spanish, Italian, and Slovak (Davies \& Cuetos, 2005; Hricová \& Weekes, 2012; Toraldo, Cattani, Zonca, Saletta, \& Luzzatti, 2006). According to the DRC model, deep dyslexia results from damage to the direct lexical pathway and nonlexical pathway so reading relies on the semantic route giving rise to semantic and visual errors (M. Coltheart, Patterson, \& Marshall, 1987). Coltheart et al., (2001) also argued that the deep dyslexia is due to atypical recruitment of the right hemisphere (see also Weekes, Coltheart \& Gordon, 1997). On 
the other hand, connectionist models of deep dyslexia (Hinton \& Shallice, 1991; Plaut $\&$ Shallice, 1993) assume that damage in the semantic reading route either in visual to semantic representations, semantic representations or semantic to phonological output gives rise to deep dyslexia. In addition, there is damage to a sublexical reading route.

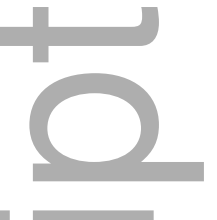

<Insert Table 6 here>

It is remarkable that Case 11 shows a pattern of reading errors that resembles surface dyslexia in other languages. Although this is expected in languages that have relatively deep orthographies, it is novel in Persian. This case had poor performance in picture naming (25\%), which may be classified as anomia, yet he could read $78 \%$ of words and $83 \%$ of nonwords correctly. As in other cases of surface dyslexia, Case 11 did not produce any semantic or mixed errors and all of his errors were formal (34 errors). Interestingly, most formal errors (27 errors) were incorrect specifications of the vowels for opaque words that are normally un-vowelized in the print (see Table 6). For example the word خرس xrs /Xers/ (bear), was read as /Xors/ (nonword), or فلف flfl /Felfel/ (pepper), was read as /FolFol/ (nonword) (See Table 6). These error types are reported in developmental dyslexia in Semitic orthographies such as Arabic and Hebrew (Friedmann \& Haddad-Hanna, 2014; Friedmann \& Lukov, 2008), presumably due to the absence of diacritics in the printed word, which then simulate the pattern of surface dyslexia. Of course, surface dyslexia in Persian must be different to patterns of surface dyslexia in other languages. For example, over dependence on reading through the non-semantic pathway (i.e. grapheme to phoneme conversion) seems to result in specifying a wrong vowel when reading opaque words (as vowels are omitted). Similar reading patterns have been reported in Arabic speaking children with developmental surface dyslexia (Friedmann \& Haddad-Hanna, 2014). These children have difficulty reading words sharing similar consonant roots but different vowels (missing in printed text). Due to the wrong specification of vowels, an unvowelized opaque word like $J M S \ddot{H}$ /jumSa/ (Friday) is read a similar word with different vowels JMS /jamaSa/ (to collect). This pattern in Hebrew is called incorrect regularization (Friedmann \& Lukov, 2008). We prefer 'wrong vowel specification' for Persian, as there is no way to determine if the patient's response errors are necessarily more regular than target words. Case 11 has a relatively severe anomia with surface dyslexia i.e. frequent wrong vowel specification for opaque 
words, a pattern which has been argued to result from diminished support from the lexico-semantic pathway (K. Patterson \& Hodges, 1992; Weekes \& Chen, 1999; Woollams, Ralph, Plaut, \& Patterson, 2007). Indeed, Woollams et al. (2007) hypothesized that for English speakers, damage to the semantic reading pathway (indeed semantic representations themselves) should produce a pattern of surface dyslexia, i.e. regularization of irregular words in English. This prediction is based on the semantic glue hypothesis first proposed by Patterson and Hodges (1992) derived from the PDP model of reading (see also Plaut et al., 1996) which holds that patients with semantic impairment such as fronto-temporal dementia (FTD) tend to rely on the nonlexical pathway for oral reading. Note there is no necessary relationship between impairment to the semantic pathway and surface dyslexia in Persian. Case 12 who was diagnosed with FTD did not show a pattern of surface dyslexia (i.e. wrong vowel specification) nor did other cases with more severe anomia (e.g. Cases 10, 14 \&16) as would be expected according to the semantic glue hypothesis. This observation fits with evidence from other languages that argues against this hypothesis as well. For example Weekes and Robinson (1997) reported a patient BP with semantic anomia who showed intact reading of low frequency exception words and poor performance in nonword reading. As BP's nonword reading was poor (which shows damage to the nonlexical reading route), they interpreted his reading as lexical non-semantic based on the DRC account. This pattern has also been observed in a Spanish speaker with semantic dementia who showed preserved performance in reading irregular borrowed words in Spanish (Wilson \& Martínez-Cuitiño, 2012) despite severe comprehension difficulties. Results here resonate with studies from other languages and argue for a direct lexical route for reading in Persian as a part of non-semantic reading pathway.

In sum, the present results suggest dissociation between lexical-semantic and nonlexical pathways for object naming and oral reading in Persian. Significant effects of different lexico-semantic variables on oral reading accuracy tends to confirm the assumption that semantic and nonsemantic routes are typically recruited during the oral reading of Persian. Furthermore, opaque words derive a benefit from semantic input and the pattern of surface dyslexia in Persian i.e. impaired vowel specification for opaque words confirms reading through a nonlexical mechanism (as a part of the non-semantic pathway) that can be error prone especially when reading opaque words in isolation. In addition, the observation of phonological dyslexia with anomia (Case 
16) suggests dissociation between semantic, lexical non-semantic and nonlexical reading pathways in Persian. The results also provide evidence of asymptomatic deep dyslexia in Persian. One limitation of the present study is a comprehensive assessment of the cognitive system related to dyslexia (spoken and written word comprehension, and phonological testing). Future research is necessary to examine mechanisms used for picture naming and oral reading more exactly in cases of aphasia in Persian.

\section{$\underline{\text { References }}$}

Alario, F. X., Ferrand, L., Laganaro, M., New, B., Frauenfelder, U. H., \& Segui, J. (2004). Predictors of picture naming speed. Behavior Research Methods, 36, 140-155.

Baayen, R. H. (2008). Analyzing linguistic data. Cambridge University Press.

Bakhtiar, M., Nilipour, R., \& Weekes, B. S. (2013). Predictors of timed picture naming in Persian. Behavior research methods, 45, 834-841.

Bakhtiar, M., \& Weekes, B. (2015). Lexico-semantic effects on word naming in Persian: Does age of acquisition have an effect? Memory \& Cognition, 43, 298-313.

Baluch, B. (1993). Lexical decisions in Persian: A test of the orthographic depth hypothesis. International Journal of Psychology, 28, 19-29.

Baluch,B., \& Danaye-Tousi, M. (2006). Spelling transparency and its impact on dyslexic and unimpaired children's memory for words. Annals of dyslexia, 56, 319-334.

Baluch, B., \& Danaye-Tousi, M. (2007). A pilot investigation into unimpaired and dyslexic Persian children's word naming and spelling: Implications for models of reading and counselling. Counselling psychology quarterly, 20, 41-50.

Baluch, B., \& Shahidi, S. (1991). Visual word recognition in beginning readers of Persian. Perceptual and Motor Skills, 72, 1327-1331.

Barry, C., \& Gerhand, S. (2003). Both concreteness and age-of-acquisition affect reading accuracy but only concreteness affects comprehension in a deep dyslexic patient. Brain and Language, 84, 84-104.

Beland, R., \& Mimouni, Z. (2001). Deep dyslexia in the two languages of an Arabic/French bilingual patient. Cognition, 82, 77-126. 
Breen, K., \& Warrington, E. K. (1995). Impaired naming and preserved reading: A complete dissociation. Cortex, 31, 583-588.

Caramazza, A. (1984). The logic of neuropsychological research and the problem of patient classification in aphasia. Brain and language, 21, 9-20.

Coltheart, M. (1978). Lexical access in simple reading tasks. In G. Underwood (Ed.), Strategies of information processing (pp. 151-216). London: Academic Press.

Coltheart, M., Curtis, B. , Atkins, P., \& Haller, M. (1993). Models of Reading Aloud: Dual-Route and Parallel-Distributed- Processing Approaches. Psychological Review, 100, 589-608.

Coltheart, M., Patterson, K., \& Marshall, J. C. . (1987). Deep dyslexia since 1980. In M. Coltheart, K. Patterson \& J. C. Marshall (Eds.), Deep dylexia. London: Routledge.

Coltheart, M., Rastle, K., Perry, C., Langdon, R., \& Ziegler, J. (2001). DRC: a dual route cascaded model of visual word recognition and reading aloud. Psychological Review, 108, 204-256.

Coltheart, V., Laxon, V. J., \& Keating, C. (1988). Effects of word imageability and age of acquisition on children's reading. British Journal of Psychology, 79, 112.

Coslett, H. B. (1991). Read but not write "idea": Evidence for a third reading mechanism. Brain and Language, 40, 425-443.

Crepaldi, D., Che, W. C., Su, I. F., \& Luzzatti, C. (2012). Lexical-semantic variables affecting picture and word naming in Chinese: a mixed logit model study in aphasia. Behavioural neurology, 25, 165-184.

Cuetos, F., Aguado, G., Izura, C., \& Ellis, A. W. (2002). Aphasic naming in Spanish: Predictors and errors. Brain and Language, 82, 344-365.

Davies, R., \& Cuetos, F. (2005). Acquired dyslexia in Spanish: A review and some observations on a new case of deep dyslexia. Behavioural neurology, 16, 85101.

Franklin, S., Howard, D., \& Patterson, K. (1995). Abstract Word Anomia. Cognitive Neuropsychology, 12, 549-566.

Friedmann, N., \& Haddad-Hanna, M. (2014). Types of developmental dyslexia in Arabic Handbook of Arabic Literacy (pp. 119-151): Springer.

Friedmann, N., \& Lukov, L. (2008). Developmental surface dyslexias. Cortex, 44, 1146-1160.

This article is protected by copyright. All rights reserved 
Frost, R. (2012). A universal approach to modeling visual word recognition and reading: Not only possible, but also inevitable. Behavioral and Brain Sciences, $35,310-329$.

Glushko, R. J. (1979). The organization and activation of orthographic knowledge in reading aloud. Journal of experimental psychology: Human perception and performance, 5, 674-691.

Gonzalez-Nosti, M., Barbon, A., Rodriguez-Ferreiro, J., \& Cuetos, F. (2014). Effects of the psycholinguistic variables on the lexical decision task in Spanish: A study with 2,765 words. Behavior research methods, 46, 517-525.

Hinton, G. E., \& Shallice, T. (1991). Lesioning an attractor network: investigations of aequired dyslexia. Psychological review, 98, 74-95.

Hirsh, K. W., \& Ellis, A. W. (1994). Age of Acquisition and Lexical Processing in Aphasia - a Case-Study. Cognitive Neuropsychology, 11, 435-458.

Hricová, M., \& Weekes, B. S. (2012). Acquired dyslexia in a transparent orthography: An analysis of acquired disorders of reading in the Slovak language.

Behavioural neurology, 25, 205-213.

Humphreys, G. W., Riddoch, M. J., \& Quinlan, P. T. (1988). Cascade Processes in Picture Identification. Cognitive Neuropsychology, 5, 67-103.

Kong, A. P., Abutalebi, J., Lam, K. S., \& Weekes, B. (2014). Executive and language control in the multilingual brain. Behavioural neurology, 2014, 527951.

Law, S. P., Weekes, B. S., Yeung, O., \& Chiu, K. (2009). Age of acquisition effects on picture naming in Chinese anomia. In S. P. Law, B. S. Weekes \& A. Wong (Eds.), Language disorders in speakers of Chinese (pp. 222-239). Clevedon: Multilingual Matters.

Law, S. P., Wong, W., \& Chiu, K. M. (2005). Preserved reading aloud with semantic deficits: evidence for a non-semantic lexical route for reading Chinese. Neurocase, 11, 167-175.

Law, S. P., Wong, W., Yeung, O., \& Weekes, B. S. (2008). A case study of the effect of age-of-acquisition on reading aloud in Chinese dyslexia. Neurocase, 14, 276-289.

Levelt, W. J. M. (1999). Models of word production. Trends in Cognitive Sciences, 3, 223-232.

Levelt, W. J. M., Roelofs, A., \& Meyer, A. S. (1999). A theory of lexical access in speech production. Behavioral and brain sciences, 22, 1-75. 
Mirdehghan, M. (2010). Persian, Urdu, and Pashto: A comparative orthographic analysis. Writing Systems Research, 2, 9-23.

Monaghan, J., \& Ellis, A. W. (2002). What exactly interacts with spelling--sound consistency in word naming? Journal of Experimental Psychology: Learning, Memory, and Cognition, 28, 183-206.

Morrison, C. M., Hirsh, K. W., Chappell, T., \& Ellis, A. W. (2002). Age and age of acquisition: An evaluation of the cumulative frequency hypothesis. European Journal of Cognitive Psychology, 14, 435-459.

New, B., Ferrand, L., Pallier, C., \& Brysbaert, M. (2006). Reexamining the word length effect in visual word recognition: new evidence from the English Lexicon Project. Psychonomic bulletin \& review, 13, 45-52.

Nickels, L. A., \& Howard, D. (1995). Aphasic naming: What matters? Neuropsychologia, 33, 1281-1303.

Nilipour, R., Pourshahbaz, A., \& Ghoreyshi, Z. S. (2014). Reliability and Validity of Bedside Version of Persian WAB. Basic and Clinical Neuroscience, 5, 253258.

Patterson, K. (2000). Phonological alexia: The case of the singing detective. In E. Funnell (Ed.), Case studies in the neuropsychology of reading (pp. 57-83). UK: Pscychology press.

Patterson, K., \& Hodges, J. R. (1992). Deterioration of word meaning: Implications for reading. Neuropsychologia, 30, 1025-1040.

Perry, C., Ziegler, J. C., \& Zorzi, M. (2007). Nested incremental modeling in the development of computational theories: the $\mathrm{CDP}+$ model of reading aloud. Psychological review, 114, 273-315.

Plaut, D. C., McClelland, J. L., Seidenberg, M. S., \& Patterson, K. (1996).

Understanding normal and impaired word reading: computational principles in quasi-regular domains. Psychological review, 103, 56-115.

Plaut, D. C., \& Shallice, T. (1993). Deep Dyslexia - a Case-Study of Connectionist Neuropsychology. Cognitive Neuropsychology, 10, 377-500.

Pouretemad, H. R., Khatibi, A., Zarei, M., \& Stein, J. (2011). Manifestations of developmental dyslexia in monolingual Persian speaking students. Archives of Iranian medicine, 14, 259-265.

Rahbari, N., \& Senechal, M. (2009). Lexical and nonlexical processes in the skilled reading and spelling of Persian. Reading and Writing, 22, 511-530. 
Rahbari, N., \& Senechal, M. (2010). Learning to read and spell in Persian: a crosssectional study from Grades 1 to 4. Developmental psychology, 46, 15141527.

Riddoch, M. J., \& Humphreys, G. W. (1993). BORB: Birmingham object recognition battery: LEA.

Seidenberg, M.S., \& McClelland, J.L. (1989). A distributed, developmental model of word recognition and naming. Psychological Review; Psychological Review, 96, 523-568.

Strain, E., Patterson, K., \& Seidenberg, M.S. (1995). Semantic effects in single-word naming. Journal of Experimental Psychology: Learning, Memory, and Cognition, 21, 1140-1154.

Toraldo, A., Cattani, B., Zonca, G., Saletta, P., \& Luzzatti, C. (2006). Reading disorders in a language with shallow orthography: A multiple single-case study in Italian. Aphasiology, 20, 823-850.

Velan, H., Frost, R., Deutsch, A., \& Plaut, D. C. (2005). The processing of root morphemes in Hebrew: Contrasting localist and distributed accounts. Language and Cognitive Processes, 20, 169-206.

Weekes, B. S. (2005). Acquired disorders of reading and writing: Cross-script comparisons. Behavioural Neurology, 16, 51-57.

Weekes, B. S. (2012). Acquired dyslexia and dysgraphia across scripts. Behavioural neurology, 25, 159-163.

Weekes, B. S., \& Chen, H. Q. (1999). Surface dyslexia in Chinese. Neurocase, 5, 161-172.

Weekes, B. S., Chen, M. J., \& Gang, Y. W. (1997). Anomia without dyslexia in Chinese. Neurocase, 3, 51-60.

Weekes, B. S., Chen, M. J., Quns, H. C., Lin, Y. B., Yao, C., \& Xiaos, X. Y. (1998). Anomia and dyslexia in Chinese: A familiar story? Aphasiology, 12, 77-98.

Weekes, B. S., Coltheart, M., \& Gordon, E. (1997). Deep dyslexia and right hemisphere reading - a regional cerebral blood flow study. Aphasiology, 11, 1139-1158.

Weekes, B. S., \& Robinson, G. (1997). Semantic anomia without surface dyslexia. Aphasiology, 11, 813-825. 
Weekes, B. S., Yin, W. G., Su, I. F., \& Chen, M. J. (2006). The cognitive neuropsychology of reading and writing in Chinese. Language and linguistics, 7, 595-617.

Wilson, M. A., \& Martínez-Cuitiño, M. . (2012). Semantic Dementia without Surface Dyslexia in Spanish: Unimpaired reading with impaired semantics. Behavioural neurology, 25, 273-284.

Woollams, A. M., Ralph, M. A. L., Plaut, D. C., \& Patterson, K. (2007). SD-squared: On the association between semantic dementia and surface dyslexia. Psychological Review, 114, 316-339.

Yang, J., McCandliss, B. D., Shu, H., \& Zevin, J. D. (2009). Simulating languagespecific and language-general effects in a statistical learning model of Chinese reading. Journal of Memory and Language, 61, 238-257.

Yin, W. G., \& Weekes, B. S. (2003). Dyslexia in Chinese: Clues from cognitive neuropsychology. Annals of Dyslexia, 53, 255-279.

Zevin, J. D., \& Seidenberg, M. S. (2002). Age of acquisition effects in word reading and other tasks. Journal of Memory and language, 47, 1-29.

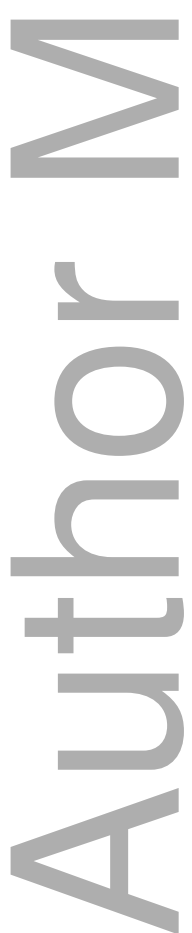




\section{University Library}

\section{- M M I E E R VA A gateway to Melbourne's research publications}

Minerva Access is the Institutional Repository of The University of Melbourne

Author/s:

Bakhtiar, M;Jafary, R;Weekes, BS

Title:

Aphasia in Persian: Implications for cognitive models of lexical processing.

Date:

2017-09

Citation:

Bakhtiar, M., Jafary, R. \& Weekes, B. S. (2017). Aphasia in Persian: Implications for cognitive models of lexical processing.. J Neuropsychol, 11 (3), pp.414-435. https://doi.org/10.1111/ jnp.12095.

Persistent Link:

http://hdl.handle.net/11343/290829 